\title{
Optimal Choice of Regularization Parameter in Image Denoising
}

\author{
Mirko Lucchese, Iuri Frosio, and N. Alberto Borghese \\ Applied Intelligent System Laboratory \\ Computer Science Dept., University of Milan \\ Via Comelico 39/41 - 20135 Milan Italy \\ \{mirko.lucchese, iuri.frosio, alberto.borghese\} @unimi.it
}

\begin{abstract}
The Bayesian approach applied to image denoising gives rise to a regularization problem. Total variation regularizers have been introduced with the motivation of being edge preserving. However we show here that this may not always be the best choice in images with low/medium frequency content like digital radiographs. We also draw the attention on the metric used to evaluate the distance between two images and how this can influence the choice of the regularization parameter. Lastly, we show that hyper-surface regularization parameter has little effect on the filtering quality.
\end{abstract}

Keywords: Denoising, Total Variation Regularization, Bayesian Filtering, Digital Radiography.

\section{Introduction}

Poisson data-noise models naturally arise in image processing where CCD cameras are often used to measure image luminance counting the number of incident photons. Photon counting process is known to have a measurement error that is modeled by a Poisson distribution [1]. Radiographic imaging, where the number of counted photons is low (e.g. a maximum count of about 10,000 photons per pixel in panoramic radiographies [2]) is one of the domains in which Poisson noise model has been largely adopted.

The characteristics of this kind of noise can be taken into account inside the Bayesian filtering framework, developing an adequate likelihood function which is, apart from a constant term, equivalent to the Kullback-Leibler (KL) divergence [3, 4]. Assuming the a-priori distribution of the solution image of Gibbs type and considering the negative logarithm of the a-posteriori distribution, the estimate problem is equivalent to a regularization problem $[5,6]$. The resulting cost function, $J($.$) , is a weighted sum of a negative log-likelihood (data-fit, J^{L}($.$) ) and a$ regularization term (associated to the a-priori knowledge on the solution, $J^{R}($.$) ).$ Tikhonov-like (quadratic) regularization often leads to over-smoothed images and Total Variation (TV) regularizers, proposed by [7] to better preserve edges, are nowadays widely adopted. As the resulting cost-function is non-linear, iterative optimization algorithms have been developed to determine the solution $[3,8]$. To get 
a differentiable cost function, a parameter, $\delta$, has been introduced into the TV term (known in this case as "hyper-surface regularizer" [11]). The regularization parameter, $\beta$, weights the two terms $J^{L}($.$) and J^{R}($.$) in J($.$) and it strongly influences the$ characteristics of the filtered image. Some attempts to set its optimal value have been proposed resorting to various forms of the discrepancy principle [9, 10], but the results are not always satisfying.

Aim of this paper is to investigate the adequacy of TV regularization in filtering radiographs and in general images with low photon counts. Results show that when low frequency components are dominant the optimal solution is obtained after a few iterations, while increasing the number of iterations the cost function further decreases but the distance between the true and the filtered image increases. We also investigated how the value of parameters $\beta$ and $\delta$ affects the filtered image and we show that the optimal value of $\beta$ is influenced by both the signal level and the frequency content of the image; on the other hand, $\delta$ has little impact: it slightly increases the regularization effect only when photon count is very low.

\section{Denoising Framework}

\subsection{Definition of the Cost Function}

Let $g_{n, j}$ and $g_{j}$ indicate respectively the gray level of the noisy and noise-free image at pixel $j$. Aim of any denoising algorithm is to estimate the true image, $\mathbf{g}=\left\{g_{j}\right\}_{j=1 . . M}$, from the measured, noisy one, $\mathbf{g}_{\mathbf{n}}=\left\{g_{n, j}\right\}_{j=1 . . M}$, where $M$ is the number of image pixels. In the Bayesian framework, the filtered image is obtained maximizing the a-posteriori probability of the image, given by the product of the likelihood of the noisy image given the filtered one, and the a-priori probability of the filtered image. Assuming that each pixel is independent from the others, and without considering the constant terms, the negative log-likelihood function can be written as:

$$
J_{\mathbf{g}_{\mathbf{n}}}^{L}(\mathbf{g})=\sum_{j=1}^{M} g_{j}-g_{n, j} \log \left(g_{j}\right),
$$

which, apart from constant terms, is equivalent to the KL divergence between $\mathbf{g}_{\mathbf{n}}$ and g. In the TV approach the regularization term is represented by the image TV norm, defined as:

$$
J^{R}(\mathbf{g})=\|\mathbf{g}\|_{T V}=\sum_{j=1}^{M} \sqrt{\left(\partial g_{j} / \partial x\right)^{2}+\left(\partial g_{j} / \partial y\right)^{2}},
$$

where $\partial g_{j} / \partial x$ and $\partial g_{j} / \partial y$ are respectively the horizontal and the vertical derivative of the image $\mathbf{g}$ in position $j$. To get a differentiable cost function even if the image gradient is equal to zero, an additional parameter, $\delta$, is introduced and the regularizer becomes:

$$
J^{R}(\mathbf{g})=\sum_{j=1}^{M} \sqrt{\left(\partial g_{j} / \partial x\right)^{2}+\left(\partial g_{j} / \partial y\right)^{2}+\delta^{2}}
$$


For $\delta=1$ the sum in (3) is equivalent to the area of the surface defined by the image values in each pixel; therefore, this is also called Hyper-surface regularization [9]. Combining eqs. (1) and (3), the following cost function is obtained:

$$
J_{\mathbf{g}_{\mathbf{n}}}(\mathbf{g})=J_{\mathbf{g}_{\mathbf{n}}}^{L}(\mathbf{g})+\beta J^{R}(\mathbf{g})=\sum_{j=1}^{N}\left[g_{j}-g_{n, j} \log \left(g_{j}\right)\right]+\beta \sum_{j=1}^{M} \sqrt{\left(\partial g_{j} / \partial x\right)^{2}+\left(\partial g_{j} / \partial y\right)^{2}+\delta^{2}}
$$

where $\beta$ is the so called regularization parameter. In the context of digital image processing, the derivatives in eq. (4) are replaced by the discrete differences between each pixel and its neighbors. In this respect, a parallel between eq. (3) and the potential function in a Markov Random Field has been drawn in [11]. In particular, let $N_{8}(j)$ be the set of indices of the eight first neighbors of $j^{\text {th }}$ pixel; eq. (4) becomes:

$$
J_{\mathbf{g}_{\mathrm{n}}}(\mathbf{g})=\sum_{j=1}^{N}\left[g_{j}-g_{n, j} \log \left(g_{j}\right)\right]+\beta \sum_{j=1}^{M} \sqrt{\sum_{i \in N_{8}}\left[\left(g_{j}-g_{i}\right) / \rho_{i, j}\right]^{2}+\delta^{2}},
$$

where $\rho_{i, j}$ is a normalization factor introduced to take into account the different distance between the $j$-th pixel and its neighbors:

$$
\rho_{i, j}=\left\{\begin{array}{cc}
\sqrt{2} & \text { if } i \text { and } j \text { are diagonal neighbors } . \\
1 & \text { otherwise }
\end{array}\right.
$$

\subsection{Minimization of the Cost Function}

The cost function in eq. (5) is strongly non linear and iterative optimization algorithms are used to minimize it. Since second order methods require the computation and inversion of the Hessian of $J_{\mathrm{gn}}(\mathbf{g})$, and since these operations are computationally intensive, minimization is usually performed via first order methods. We use here the recently proposed Scaled Gradient Projection method [4] that has shown fast convergence rate. At each step, the solution is updated as:

$$
\mathbf{g}^{k+1}=\mathbf{g}^{k}+\lambda_{k} \mathbf{d}^{k},
$$

where $\mathbf{d}^{k}$ is a descent direction derived from the Karush - Kuhn - Tucker conditions, and $\lambda_{k}$ is determined such that the decrease of the solution is guaranteed to be large along that direction. The procedure is stopped when the normalized variation of the cost function goes below a given threshold $\tau$, that is:

$$
\left|\left[J_{\mathbf{g}_{\mathbf{n}}}\left(\mathbf{g}^{k+1}\right)-J_{\mathbf{g}_{\mathbf{n}}}\left(\mathbf{g}^{k}\right)\right] / J_{\mathbf{g}_{\mathbf{n}}}\left(\mathbf{g}^{k+1}\right)\right| \leq \tau,
$$

or when the maximum number of iterations is achieved.

\section{Experimental Setup}

We have created a set of simulated digital radiographs of $512 \times 512$ pixels as follows. First, an absorption coefficients map was created, with coefficients increasing from $0 \%$ for the left-most pixels to $100 \%$ for the right-most ones. Then, 50 different geometrical figures (circles and rectangles) were randomly positioned inside the image. The radius of the circles and the rectangle sides had length randomly chosen between 1 and 512 pixels. Each time a circle or a rectangle was added to the map, all 
the absorption coefficients covered by the figure were modified: either they were substituted by their complements with respect to $100 \%$, or they were multiplied by a random value between 0 and 1 , or a random value between $0 \%$ and $100 \%$ was added to them. In the latter case, the resulting absorption coefficients were always clipped to $100 \%$. The choice among the three modalities was random. To control the frequency content, these images were filtered with different moving average filters with size of $41 \times 41,23 \times 23$ and $9 \times 9$ pixels to generate respectively low (LF), medium (MF) and high frequency $(\mathrm{HF})$ simulated radiographs. Three sets of images were considered each with a different maximum number of photons reaching the sensor: 10,000, 1,000 and 100 photons. For each image, we considered five realizations of Poisson noise; we explicitly notice that the images with low photon count have noise with a lower standard deviation (and lower signal to noise ratio, SNR) than those at high photon count due to the nature of Poisson noise. Three typical images are shown in Fig. 1.

We filtered the noisy images with the method in Section 2 and we measured the difference between each filtered image and the true one using three different quality indices. The first one is the Root Mean Squared Error (RMSE), which is widely adopted in signal and image processing as it is related to the power of the error:

$$
\operatorname{RMSE}\left(\mathbf{g}_{1}, \mathbf{g}_{2}\right)=\sqrt{\sum_{j=1}^{M}\left(g_{1, j}-g_{2, j}\right)^{2} / M} .
$$

The second index is the Structural Similarity (SSIM) proposed in [12]. This index compares local patterns of pixel intensities that have been normalized for luminance and contrast and it evaluates the similarity by comparing structural information from processed images. It is defined as:

$$
\operatorname{SSIM}\left(\mathbf{g}_{1}, \mathbf{g}_{2}\right)=\frac{1}{M} \sum_{j=1}^{M}\left[\left(\frac{2 \mu_{\mathbf{g}_{1}}(j) \cdot \mu_{\mathbf{g}_{2}}(j)+c_{1}}{\mu_{\mathbf{g}_{1}}^{2}(j)+\mu_{\mathbf{g}_{2}}^{2}(j)+c_{1}}\right) \cdot\left(\frac{2 \operatorname{cov}_{\mathbf{g}_{1}, \mathbf{g}_{2}}(j)+c_{2}}{\sigma_{\mathbf{g}_{1}}^{2}(j)+\sigma_{\mathbf{g}_{2}}^{2}(j)+c_{2}}\right)\right],
$$

where $\mu_{\mathrm{g}}(j)$ is the local mean of $\mathbf{g}$ in the neighborhood of the $j$-th pixel, $\operatorname{cov}_{\mathrm{g} 1, \mathrm{~g} 2}(j)$ is the local covariance between $\mathbf{g}_{1}$ and $\mathbf{g}_{2}, \sigma_{\mathrm{g}}^{2}(j)$ is the local variance of the image $\mathbf{g}$ and the constants $c_{1}$ and $c_{2}$ are defined as $c_{1}=\left(k_{1} L\right)^{2}$ and $c_{2}=\left(k_{2} L\right)^{2}$ where $L$ is the maximum image gray level (e.g. 255 for 8 bit images) and $k_{1}$ and $k_{2}$ are user defined constants set equal to 0.01 and 0.03 according to [12].

The last index is the Features Similarity (FSIM) proposed in [13]. This index, based on the phase congruency (PC) model of the Human Visual System (HVS), takes into account the local phase of the Fourier components of the image and the local gradient magnitude. It is defined as:

$$
\operatorname{FSIM}\left(\mathbf{g}_{1}, \mathbf{g}_{2}\right)=\sum_{j=1}^{M} w(j)\left[\left(\frac{2 P C_{\mathbf{g}_{1}}(j) \cdot P C_{\mathbf{g}_{2}}(j)+T_{1}}{P C_{\mathbf{g}_{1}}^{2}(j)+P C_{\mathbf{g}_{2}}^{2}(j)+T_{1}}\right) \cdot\left(\frac{2 G_{\mathbf{g}_{1}}(j) \cdot G_{\mathbf{g}_{2}}(j)+T_{2}}{G_{\mathbf{g}_{1}}^{2}(j)+G_{\mathbf{g}_{2}}^{2}(j)+T_{2}}\right)\right],
$$

where $\mathrm{PC}_{\mathbf{g}}$ is the phase congruency term and $\mathrm{G}_{\mathbf{g}}$ is the gradient magnitude in $j$, computed here by Scharr operator. $\mathrm{T}_{1}$ and $\mathrm{T}_{2}$ are user defined constants and, for 8-bit images, they are equal to 0.85 and 160 respectively [13]. In our case $\mathrm{T}_{2}$ was linearly rescaled proportionally to the maximum gray levels. The weights $w(j)$ are computed to assign a higher weight to the position with high phase congruency [13]: 


$$
w(j)=P C_{m}(j) / \sum_{j=1}^{M} P C_{m}(j),
$$

where $\mathrm{PC}_{\mathrm{m}}(j)=\max \left\{\mathrm{PC}_{\mathrm{g}_{1}}(j), \mathrm{PC}_{\mathbf{g} 2}(j)\right\}$.

The difference between the filtered and true image has been evaluated for different values of $\beta$ (in the range from 0.01 to 0.9 ) and for $\delta=\left\{0\right.$, eps $=2.2204 \times 10^{-16}, 0.01$, $0.05,0.1,0.5,1\}$. To avoid singular derivatives of $J^{R}(\mathbf{g})$ for $\delta=0, \partial J^{R}(\mathbf{g}) / \partial g_{j}$ was assumed equal to zero for all the pixels $j$ whose gradient norm was equal to zero.
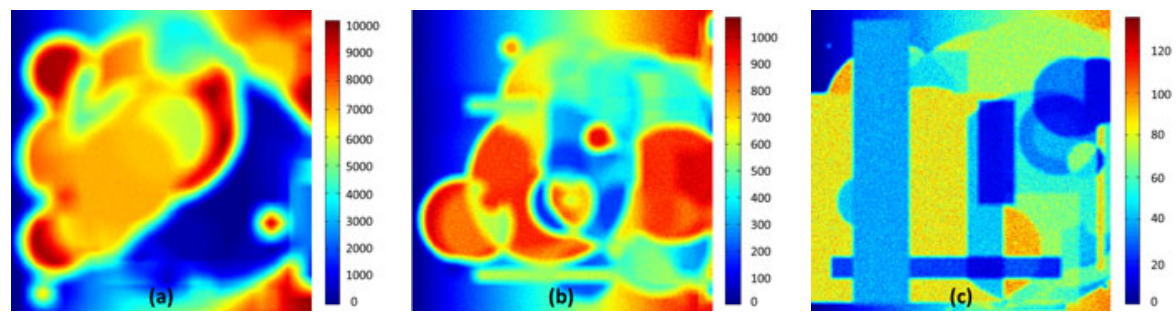

Fig. 1. Panels (a-c) show a LF, MF and HF simulated radiograph whose expected maximum number of photons is equal to, respectively, 10,000, 1,000 and 100

\section{Results and Discussion}

Figs. 2, 3 and 4 represent the mean value of RMSE, SSIM and FSIM respectively, averaged over five filtered images, as a function of $\beta$ and $\delta$. The optimal value of $\beta$ is higher for LF images and lower for MF and HF images (Fig. 5): in practice, when the structures (edges) in the image become sharper, the regularizer should be more edgepreserving. This effect is obtained lowering the value of $\beta$ in the cost function (5), and therefore increasing the probability of observing high gradients (associated to edges) in the filtered image. The same figures show that the optimal value of $\beta$ increases when the number of photons decreases. In fact, according to Poisson statistics, the SNR is low when the number of counted photons is low: in this case, the low reliability of the measured data has to be counterbalanced by a high regularization and therefore it calls for high values of $\beta$.

Figs. 2-4 also show also that RMSE generally leads to an optimal value of $\beta$ lower than that suggested by SSIM and FSIM, although the values provided by FSIM and by RMSE are very similar in almost all cases. This reflects different capacities of the indices to quantify the image quality. More in detail, the visual inspection of the images filtered with the optimal $\beta$ value suggested by different indices reveals that the SSIM generally provides a too regular image, especially when the photon count is low (Fig 6). Overall, although SSIM has been proposed as a principled evaluation metric based on the properties of the HVS, RMSE generally provides a less regularized image with lower noise and more visible edges. In case of low photon count images, FSIM shows a higher capability of identifying the optimal value of $\beta$ than SSIM (curves in Fig. 4g-i have higher curvature than those in Fig. 3h-i), providing results similar to RMSE. Visual inspection of filtered images with low photons count reveals that images suggested as optimal by RMSE and FSIM definitely appear more similar to the ground truth than the image obtained with SSIM (Fig. 6). 

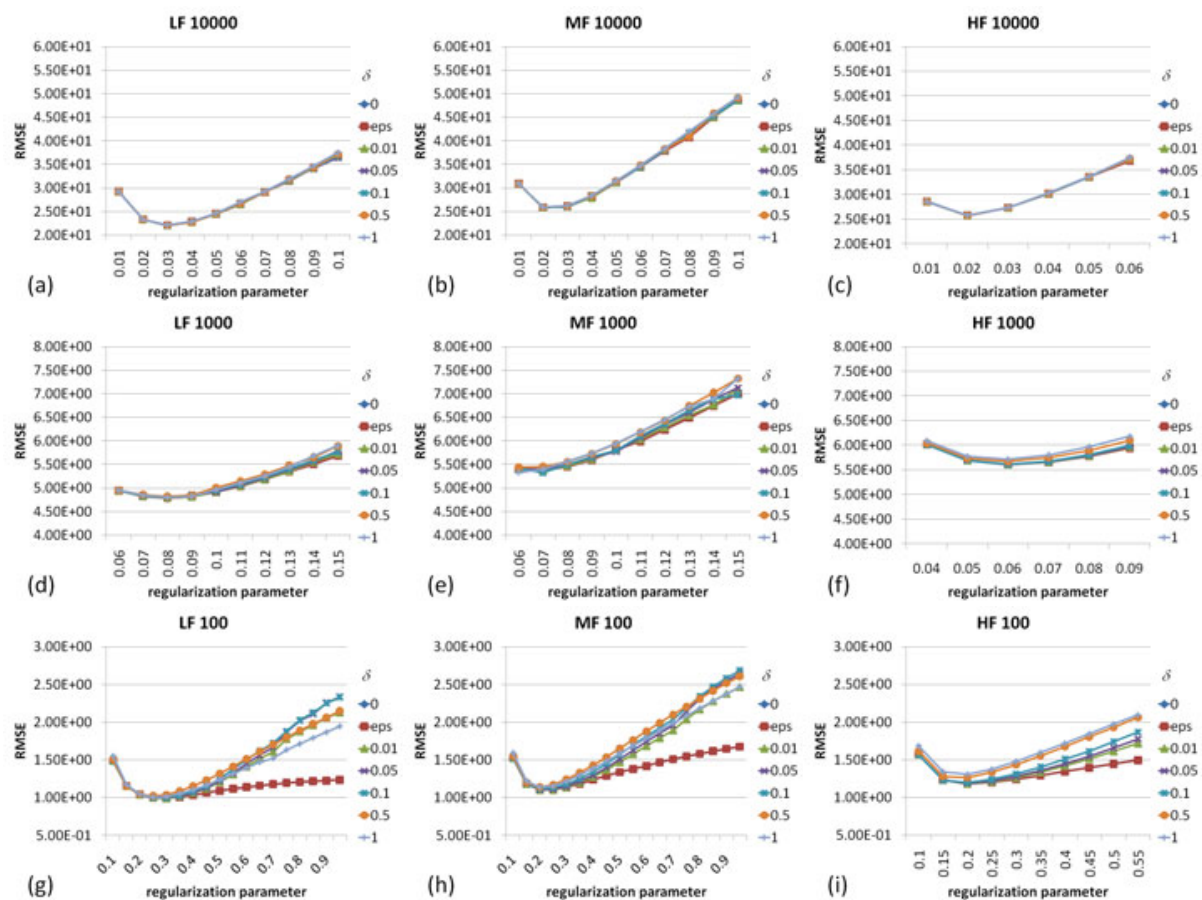

(c)

$\begin{array}{llllll}0.01 & 0.02 & 0.03 & 0.04 & 0.05 & 0.06\end{array}$
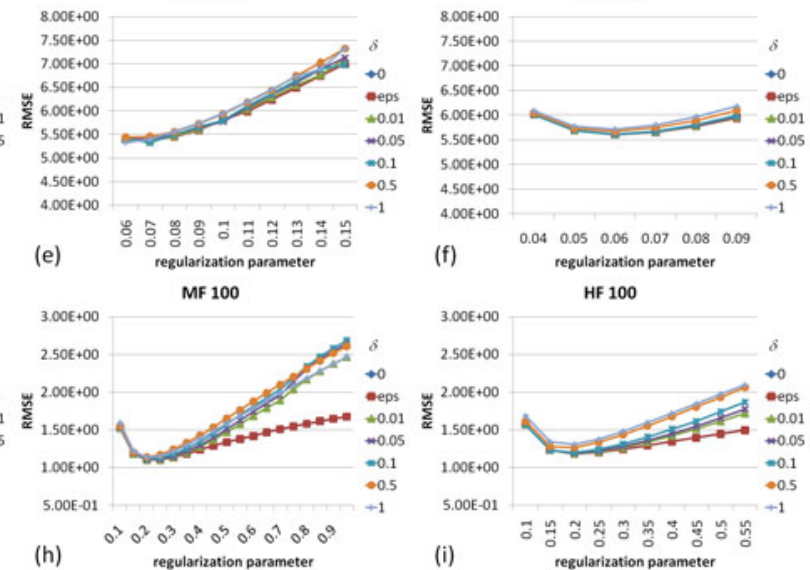

Fig. 2. Mean RMSE as a function of the regularization parameter $\beta$. Here, eps $=2.2204 \times 10^{-16}$. The title of each graph reports the frequency content of the images and the number of photons.

The similarity indices in figs. 2, 3 and 4 also indicate that the parameter $\delta$ has little or even no influence on the quality of the filtered image for medium and high photon counts as far as it assumes a very small value; in fact, the curves reported in these figures show low dependency on the value of $\delta$. For a low number of photons, better results are obtained with small values of $\delta$ ( 0 or eps). This fact is explained considering that, for low counts, the term $\left[\left(g_{i}-g_{i}\right) / \rho_{i, j}\right]^{2}$ in the TV norm in eq. (5) is low and therefore $\delta$ may strongly influence the value of the TV norm.

For LF and MF images, optimization exhibits semi-convergence: the similarity between the filtered image and the ground truth increases in the first iterations, reaches a maximum and then decreases (Fig. 7a-b). This is not the case of HF images, at least up to the $800^{\text {th }}$ iteration (Fig. 7c-d). This fact can be explained considering that TV regularization implicitly assumes that images are composed by flat areas separated by sharp edges. However LF and MF images, that are somehow similar to digital radiographies, are not coherent with such hypothesis. As a result, the filter cuts the image valleys and ridges introducing spurious plateaus in the high photon count areas; the cost function is actually minimized but the image is over-smoothed (Fig. 8b). This phenomenon becomes evident only in the last iterations and it is less evident in the low photon count regions. To analytically explain it, let us consider the derivative of the likelihood term in eq. (1): 


$$
\partial J_{\mathbf{g}_{\mathbf{n}}}^{L}(\mathbf{g}) / \partial g_{j}=1-g_{n, j} / g_{j} .
$$

Defining $\Delta g_{j}=g_{j}-g_{n, j}$, eq. (16) becomes:

$$
\partial J_{\mathbf{g}_{\mathbf{n}}}^{L}(\mathbf{g}) / \partial g_{j}=1-g_{n, j} /\left(g_{n, j}+\Delta g_{j}\right) .
$$

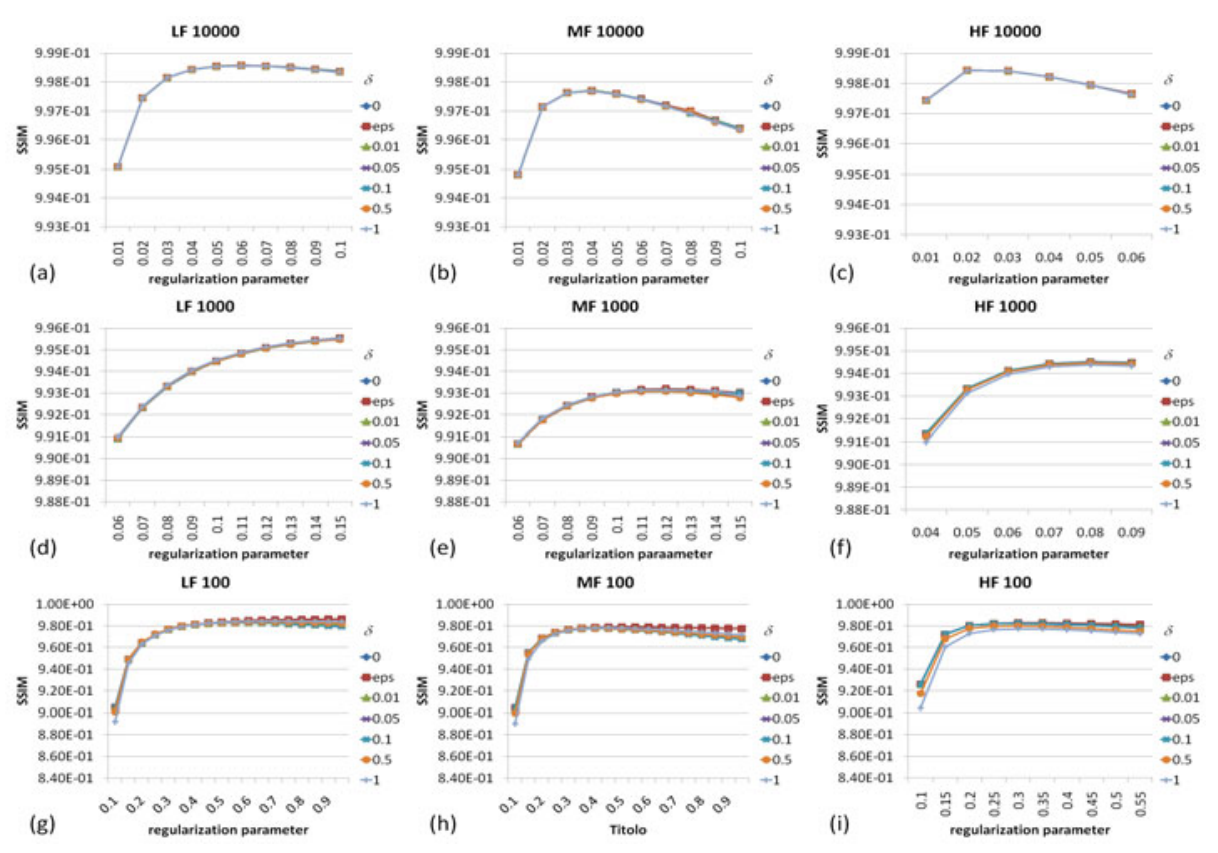

Fig. 3. Mean SSIM index varying the regularization parameter $\beta$. Here, eps $=2.2204 \times 10^{-16}$. The title of each graph reports the frequency content of the images and the number of photons.

Since $g_{n, j}$ is fixed, eq. (17) approximately expresses the variation of the likelihood as a function of $\Delta g_{j}$. If the noisy gray level, $g_{n, j}$ is high, $\partial J_{\mathbf{g}_{\mathbf{n}}}^{L}(\mathbf{g}) / \partial g_{j}$ is close to zero for large intervals of $\left|\Delta g_{j}\right|$. In other words, the $j^{\text {th }}$ element of the likelihood in (5) slightly differs from its minimum even if the filtered image significantly differs from the noisy one. In these regions, the filtering effect depends mainly on the regularization term, which tends to suppress the edges, introducing a plateau where a ridge (or a valley) is present (Fig. 8b). On the other hand, if $g_{n, j}$ is small, a high value of $\left|\Delta g_{j}\right|$ produces a significant increase of $J_{\mathbf{g}_{\mathbf{n}}}^{L}(\mathbf{g})$. In this case, the variation of the cost function is mainly influenced by the likelihood which constrains the solution to be close to the noisy image, thus retaining noise oscillations (Fig. 8d). This further explains the need of a higher value of $\beta$ for low-photon count images: in this case the SNR is low, the measured values are less reliable, $\left|\partial J_{\mathbf{g}_{\mathbf{n}}}^{L}(\mathbf{g}) / \partial g_{j}\right|$ increases significantly for small $\left|\Delta g_{j}\right|$ and more regularization is necessary to get the denoising effect. 


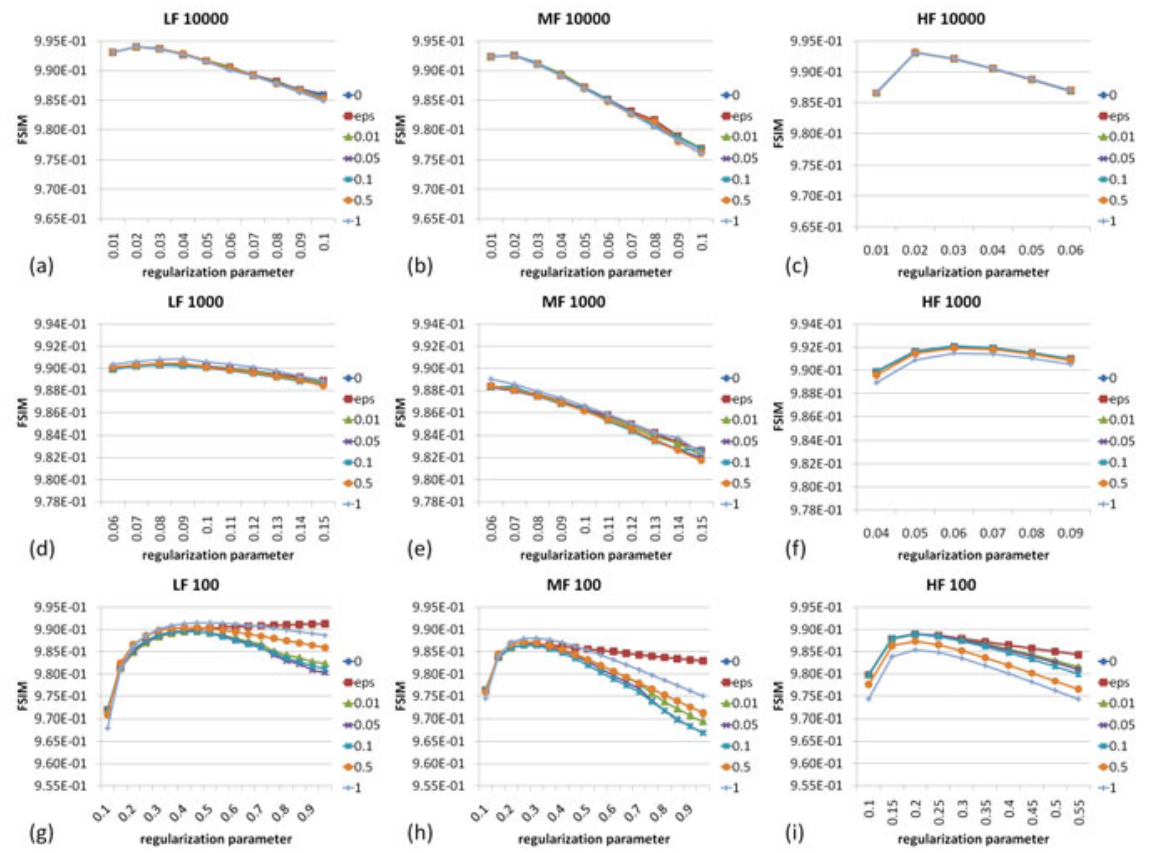

Fig. 4. Mean FSIM index varying the regularization parameter $\beta$. Here, eps $=2.2204 \times 10^{-16}$. The title of each graph reports the frequency content of the images and the number of photons.
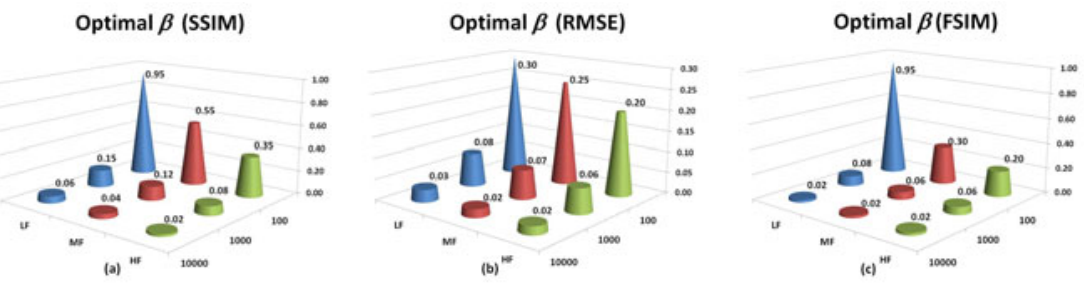

Fig. 5. Optimal values of $\beta$ associated to the optimal value of SSIM (a), RMSE (b) and FSIM (c) as a function of photon count and image frequency content
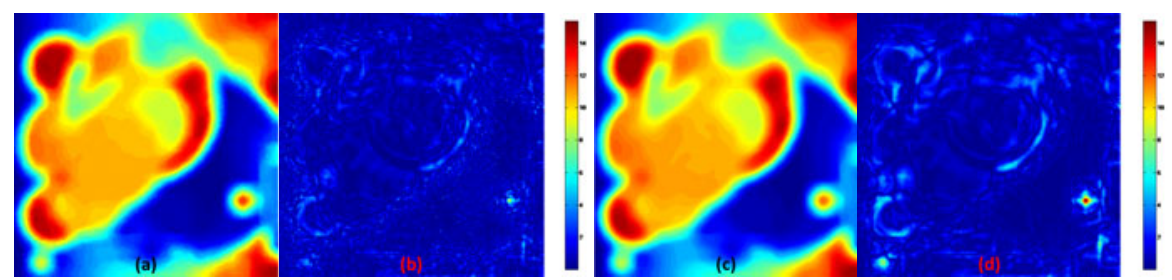

Fig. 6. Filtered LF 100 image with $\delta=$ eps and $\beta=0.3$ (a) and $\beta=0.95$ (c), corresponding to the optimal value suggested by RMSE and SSIM respectively. Panels (b) and (d) show the absolute difference between the filtered image and the ground truth. 

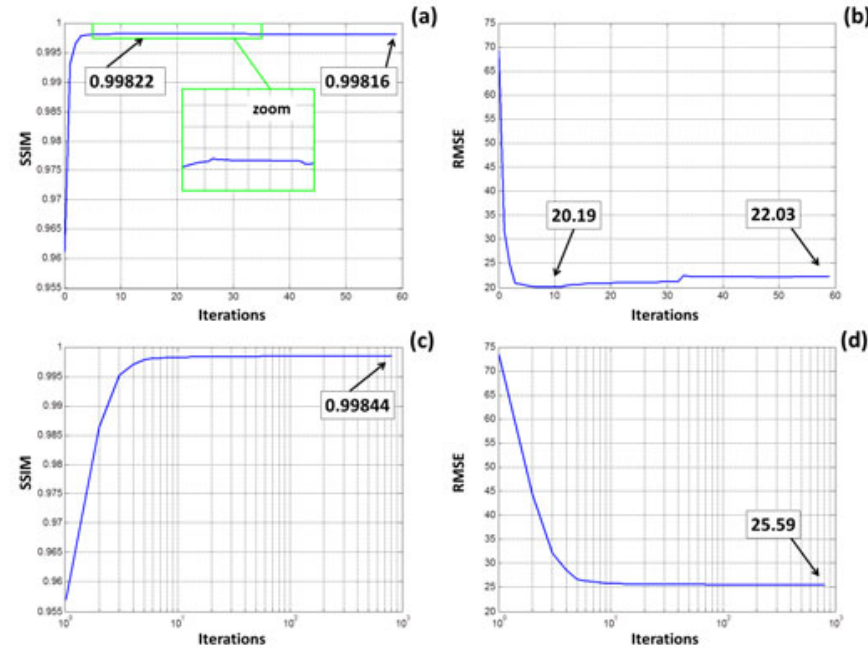

Fig. 7. In (a) and (b), SSIM and RMSE are reported for a LF 10,000 image versus the iteration number. The same indexes are reported for a HF 10,000 image in (c) and (d), for $\tau=10^{-14}$. Panel (a) includes a zoom of the area where SSIM stops increasing (semi-convergence). Numbers in the panels show the index value at the iteration indicated by the corresponding arrow.
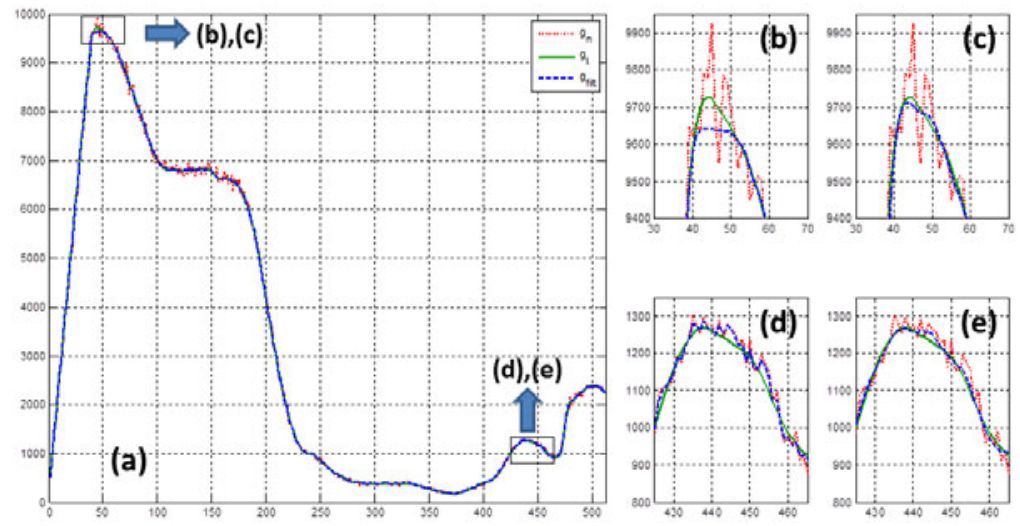

Fig. 8. The true profile of one row of a LF 10,000 image is shown in green in panel (a); the blue line is the filtered $\left(\beta=0.03\right.$ and $\tau=10^{-10}$ ) profile, the red line represents the noisy one. Panels (b) and (d) show zooms of ridges with high and low photon counts. Panels (c) and (e) show the same areas of (b) and (d), when the filtered image has been obtained with $\beta=0.64$ and $\tau=10^{-4}$, corresponding to only four iterations.

Plateau introduction in LF and MF images is prevented by small number of iterations (e.g. $\tau=10^{-4}$ ) and high value of $\beta$. The ridges in high photon counts areas are preserved by the low number of iterations; the high value of $\beta$ gives the proper weight to the regularization term in the low photon count areas. This definitely produces an image with effective denoise in the low count regions (Fig. 8e) without cutting ridges and valleys (Fig. 8c) in the high count areas. 


\section{Conclusion}

Image denoising through TV regularization is nowadays widely diffused as a "Holy Graal" of image filtering. However, as shown here, it may not be the best choice, at least for digital radiographs. In fact, the semi-convergence property observed for $\mathrm{MF}$ and LF images suggests the need for developing a better model for a-priori term, at least for this kind of images. On the other hand, also the definition of a general, reliable image quality index remains an open issue, as demonstrated by the difference in the optimal value of $\beta$ obtained when RMSE, SSIM or FSIM measure were used to evaluate the difference between the filtered and the true image.

\section{References}

1. Snyder, D.L., Hammoud, A.M., White, R.L.: Image recovery from data acquired with a charge coupled device camera. J. Opt. Soc. Am. A 10, 1014-1023 (1993)

2. Frosio, I., Borghese, N.A.: Statistical Based Impulsive Noise Removal in Digital Radiography. IEEE Trans. on Med. Imag. 28(1), 3-16 (2009)

3. Zanella, R., Boccacci, P., Zanni, L., Bertero, M.: Efficient Gradient Projection methods for edge-preserving removal of Poisson noise. Inverse Problems 25(4), 045010 (2009)

4. Bonettini, S., Zanni, L., Zanella, R.: A scaled gradient projection method for constrained image deblurring. Inverse Problems 25(1), 015002 (2009)

5. Bertero, M., Lanteri, H., Zanni, L.: Iterative image reconstruction: a point of view. In: Proc. IMRT (2008)

6. Tikhonov, A.N., Arsenin, V.Y.: Solutions of Ill-posed Problems. W. H. Winston (1977)

7. Rudin, L., Osher, S., Fatemi, E.: Nonlinear Total Variation based noise removal algorithms. Physica D 60, 259-268 (1992)

8. Carbonetto, P., Schmidt, M., de Freitas, N.: An interior-point stochastic approximation method and an L1-regularized delta rule. In: Proc. NIPS 2008, pp. 112-119 (2008)

9. Bertero, M., Boccacci, P., Talenti, G., Zanella, R., Zanni, L.: A discrepancy principle for Poisson data. Inverse Problems 26(10), 105004 (2010)

10. Lucchese, M., Borghese, N.A.: Denoising of Digital Radiographic Images with Automatic Regularization Based on Total Variation. In: Proc. ICIAP, pp. 711-720 (2009)

11. Geman, S., Geman, D.: Stochastic Relaxation, Gibbs Distributions, and the Bayesian Restoration of Images. IEEE Trans. PAMI 6, 721-741 (1984)

12. Wang, Z., Bovick, A.C., Sheikh, H.R., Simoncelli, E.P.: Image Quality Assessment: From Error Visibility to Structural Similarity. IEEE Tran. Image Proc. 13(4), 600-612 (2004)

13. Zhang, L., Zhang, L., Mou, X., Zhang, D.: FSIM: A Feature Similarity Index for Image Quality Assessment. IEEE Tran. Image Proc. (in press) 